\title{
Lymphatic mapping and sentinel node
} biopsy in endometrial cancer - a feasibility study using cervical injection of radiotracer and blue dye

Sima Kadkhodayan', Zahra Shiravani', Malihe Hasanzadeh ${ }^{1}$, Nourieh Sharifi', Zohreh Yousefi', Asiehsadat Fattahi ${ }^{3}$, Ramin Sadeghi ${ }^{4}$

'Women's Health Research Center, Mashhad University of Medical Sciences, Mashhad, Iran ${ }^{2}$ Pathology Department, Ghaem Hospital, Mashhad University of Medical Sciences, Mashhad, Iran ${ }^{3}$ Minimally Invasive and Endoscopic Surgery Research Center, Mashhad University of Medical Sciences, Mashhad, Iran ${ }^{4}$ Nuclear Medicine Research Center, Mashhad University of Medical Sciences, Mashhad, Iran

The authors declare no conflict of interest.

Acknowledgements: This study is the result of a residency thesis under the approval number of ${ }^{89706}$, and was conducted in the Nuclear Medicine and Women's Health Research Centers of Mashhad University of Medical Sciences. The study was supported financially by the vice chancellery of research of Mashhad University of Medical Sciences.

[Received 30 X 2013; Accepted 7 VII 2014]

\begin{abstract}
BACKGROUND: The aim of this study was to evaluate the feasibility and accuracy of sentinel lymph node (SLN) detection using preoperative lymphoscintigraphy and intra-operative gamma probe/blue dye for endometrial cancer patients.

MATERIAL AND METHODS: Twenty four consecutive patients with endometrial cancer were recruited. All patients underwent lymphatic mapping and sentinel node biopsy using combined intracervical radiotracer and blue dye injections. Pelvic lymph node dissection was performed for all patients. Para-aortic lymphadenectomy was done in high risk patients. All SLNs were examined by frozen section and Hematoxylin and Eosin (H\&E) permanent sections.

RESULTS: Pre-operative lymphoscintigraphy showed at least one SLN in 21/24 patients. Intra-operatively, at least one SLN could be harvested by gamma probe and/or blue dye methods. A total of 95 SLNs were detected. Four SLNs were detected only by blue dye, 42 only by radiotracer, and 49 were hot/blue. Median number of SLN per patient was 3 . Three patients had positive pelvic lymph nodes. All of them had positive SLN (no false negative case). Frozen section could identify SLN involvement in two of three patients with positive pathology.

CONCLUSION: Lymphatic mapping and sentinel node biopsy is feasible and accurate in endometrial cancer patients using combined radiotracer and blue dye methods. Frozen section accuracy was lower and underscores the importance of expert pathologists for SLN mapping technique.
\end{abstract}

KEY words: sentinel node, endometrial cancer, blue dye, cervical injection, radiotracer, endometrium, uterine

Nuclear Med Rev 2014; 17, 2: 55-58

Correspondence to: Sadeghi Ramin, MD

Nuclear Medicine Research Center, Mashhad University of Medical

Sciences, Mashhad, Iran

Phone: +985118012202

Fax: +985118933186

E-mail: sadeghir@mums.ac.ir; raminsadeghi1355@yahoo.com

\section{Background}

Gynecological cancers are important female life-threatening malignancies among which endometrial cancer is the most common in most areas of the world [1]. The primary surgical procedure for endometrial cancer patients include total hysterectomy with bilateral salpingo-oophorectomy [2]. A controversial issue in the 
management of the endometrial cancer is whether or not to perform systematic pelvic lymphadenectomy and paraaortic lymphadenectomy because of the inability to predict those patients who would benefit from lymph node dissection [3-5].

Complete pelvic and paraaortic lymphadenectomy may cause severe surgical complications which can considerably affect the quality of life of the patients [6]. In order to avoid lymphadenectomy, the sentinel lymph node (SLN) concept has been applied to the treatment of several solid tumors [7-9]. This concept has also been used in endometrial cancer patients since 1996 and many groups reported their experience on this technique thus far $[10,11]$.

The aim of this study was to evaluate the feasibility and accuracy of sentinel lymph node detection using preoperative lymphoscintigraphy and intra operative gamma probe/blue dye for endometrial cancer patients.

\section{Material and methods}

From October 2010 to December 2012, consecutive patients who had a histologically verified endometrial cancer and early stage on pre-operative evaluation underwent lymphatic mapping and were enrolled into the study. The study was approved by the ethics committee of Mashhad University of Medical Sciences under the approval number of 89706 and all patients were required to sign an informed consent. The procedures followed were in accordance with the Helsinki Declaration.

Preoperative lymphoscintigraphy was obtained following intracervical injection (at 3 and 9 o'clock positions) of 2 mCi radiotracer (Tc-99m-Antimony Sulfide Colloid in 15 patients and Tc-99m-Phytate in 9 patients) in $0.4 \mathrm{~mL}$ volume divided to two aliquots. Imaging was done 15 minutes post-radiotracer injection (ANT-POST and Lat views $5 \mathrm{~min} /$ view using low energy high resolution collimator and Tc-99m photopeak) with a dual head variable angle gamma camera (E.CAM, Siemens) $[12,13]$.

The day after radiotracer injection (18-24 hours post injection), the patients underwent surgical operation. In all patients, methylene blue dye was injected directly into the cervix in the operating room. The injection was performed just before incising the skin. Two $2 \mathrm{ml}$ syringes, each containing methylene blue dye $(0.5 \mathrm{ml})$ were used for each patient. Injection sites were the same as the radiotracer.

Detection of SLNs was accomplished through direct visualization of blue colored lymphatics and nodes, and/or by detection of radioactivity, using a handheld gamma probe (EUROPROBE, France). All hot and/or blue nodes were harvested and sent for intra-operative frozen section evaluation. The surgeon then proceeded to do a complete pelvic lymphadenectomy in all cases regardless of the SLNs frozen section results. Para-aortic lymphadenectomy was done only in selected cases (clear cell, serous or adenosquamous types, grade 2 or 3 endometrioid carcinomas). Bilateral lymphadenectomy was followed by total hysterectomy and bilateral salpingo-oophorectomy.

\section{Results}

Overall, 24 patients were included in the study. Table 1 shows the characteristics of the included patients. All patients were injected with both radiotracer and blue dye. However, gamma probe was defective during surgery of one patient and only blue dye technique was used for this specific patient.
Table 1. Characteristics of the study population

\begin{tabular}{|c|c|}
\hline Age (years) & $56(33-86)$ \\
\hline $\mathrm{BMI}\left[\mathrm{kg} / \mathrm{m}^{2}\right]$ & $32(25-35)$ \\
\hline \multicolumn{2}{|l|}{ Histology } \\
\hline Endometrioid & 20 \\
\hline Papillary serous & 3 \\
\hline Clear cell & 1 \\
\hline \multicolumn{2}{|l|}{ Grade } \\
\hline । & 9 \\
\hline ॥ & 7 \\
\hline III & 4 \\
\hline \multicolumn{2}{|l|}{ Stage } \\
\hline 1 & 14 \\
\hline ॥ & 4 \\
\hline III & 5 \\
\hline IV & 1 \\
\hline \multicolumn{2}{|l|}{ Lymphovascular invasion } \\
\hline Present & 9 \\
\hline Absent & 15 \\
\hline \multicolumn{2}{|l|}{ Myometrial invasion } \\
\hline$<1 / 2$ & 10 \\
\hline$>1 / 2$ & 14 \\
\hline \multicolumn{2}{|l|}{ Lymph node surgery } \\
\hline Pelvic lymph node dissection & 10 \\
\hline \multicolumn{2}{|l|}{ Pelvic and para-aortic lymph } \\
\hline node dissection & 14 \\
\hline
\end{tabular}

Preoperative lymphoscintigraphy visualized SLN in 21/24 patients (Figure 1). Intraoperative localization of the SLN was possible in all 24 patients (100\% surgical detection rate). A total of 95 SLNs were identified (median SLNs per patient of 3). SLNs were detected bilaterally in 16 and unilaterally in 8 patients. The anatomic distribution of the SLNs is shown in Table 2. No para-aortic SLN was detected intra-operatively. Four SLNs were detected only by blue dye, 42 only by radiotracer, and 49 were hot/blue.

Pelvic lymph nodes were pathologically involved in 3 patients. SLNs of these three patients were also positive on pathological examination ( 2 were positive by frozen section and 1 by H\&E examination). The false negative rate for $\mathrm{H} \& \mathrm{E}$ was $0 \%$ and for frozen section examination was 33\%. Two of these patients had pathologically involved non-SLN too.

\section{Discussion}

Our results revealed a 100\% detection rate, suggesting that SLN detection is feasible using intra-operative and preoperative cervical injections for endometrial cancer. Furthermore, SLN detection was not related to histological type or grade of endometrial carcinoma. Preoperative lymphoscintigraphy with cervical injection of radiotracer visualized SLN in 21/24 patients. More importantly, intraoperative localization of the SLN was possible in all patients even those with lymphoscintigraphy detection failure. In another study, Abu-Rustum et al. reported that preoperative lymphoscintigraphy visualized SLN in 30 


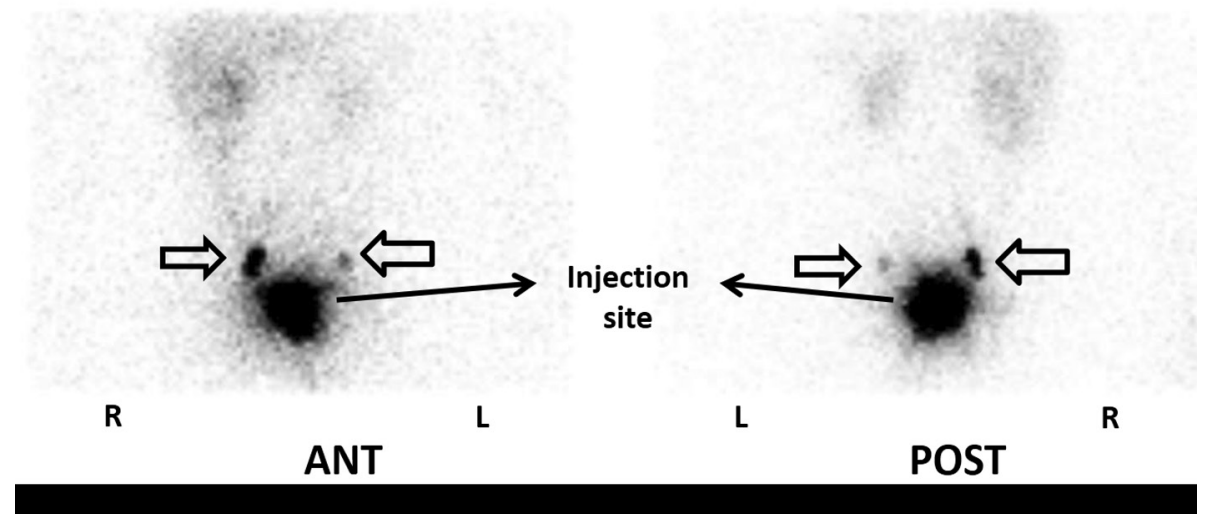

Figure 1. Anterior-Posterior lymphoscintigraphy images of a patient after cervical injection of the radiotracer. The sentinel nodes are shown in open arrows. At surgery two hot/blue external iliac nodes on the right side and one hot internal iliac node on the left side were detected

Table 2. Anatomical distribution of the SLNs

$\begin{array}{lc}\text { Anatomic sites of SLN } & \text { Number of SLNs } \\ \text { External iliac } & \\ \text { Right } & 12 \\ \text { Left } & 7 \\ \text { Internal iliac } & \\ \text { Right } & 16 \\ \text { Left } & 13 \\ \text { Obturator } & \\ \text { Right } & 26 \\ \text { Left } & 15 \\ \text { Common iliac } & \\ \text { Right } & 5 \\ \text { Left } & 1\end{array}$

out of 42 endometrial cancer patients. Intraoperative localization of the SLN was successful in 36 patients [14]. Niikura et al. also reported the same findings as lymphoscintigraphic SLN detection was 68\% despite $82 \%$ detection rate at surgery [15]. The above-mentioned findings can be due to proximity of injection site to the lymphatic basin which can mask the sentinel nodes on lymphoscintigraphy images [16, 17]. This problem can be overcome by SPECT/CT to some extent [18-20]. By improving the localization ability of the nuclear medicine imaging, SPECT/CT can contribute a lot to the sentinel node mapping of gynecological cancers. Lymphatic flow to the unusual anatomical sites can be also detected by SPECT/CT. It seems that SPECT/CT is becoming an integral part of sentinel node mapping in surgical oncology.

The mapping method was highly related to SLN detection rate in our study, as the majority of harvested SLNs were detected by radiotracer and contribution of blue dye was minimal. The blue only SLNs were found during surgery of a patient without gamma probe at hand. Several other reports also supported our findings as detection rate using radiotracer or combined radiotracer and blue dye was higher than blue dye alone [21-24]. Considering the potential risks of blue dye injections (such as life threatening anaphylaxis reactions), radiotracer alone may be sufficient for SLN mapping in endometrial cancer [25].
An important issue in the SLN mapping of endometrial cancer is the injection site of the mapping material. We used cervical injection technique which is much easier than the fundal or sub-endometrial injections. High detection rate and sensitivity of our study was in accordance to other studies used cervical injection $[19,26]$. Cervical injection is associated with extremely low para-aortic lymphatic drainage and some groups argue against this technique due to this fact. For example Solima et al. reported that $56 \%$ of their patients had para-aortic SLNs using sub-endometrial injection of the radiotracer [27]. However, frequency of isolated para-aortic without pelvic lymph node involvement in endometrial cancer patients is reported to be low. This was only $2 \%$ in Abu-Rustam et al. study [28]. We didn't have any para-aortic node involvement in the patients underwent para-aortic lymph node dissection.

False negative rate is the most important outcome measure in all SLN mapping studies. Two recent systematic reviews evaluated the sentinel node concept in endometrial cancer and showed sensitivity about $90 \%$ or more $[23,29]$. We didn't have any false negative results in our study either, as all 3 patients with involved pelvic nodes had positive SLNs too. However, the frozen section accuracy was lower with 1 (33\%) false negative results. This false negative case occurred in the beginning of our study and can be attributed to the learning curve effect. This finding underscores the importance of expert pathologist in the evaluation of SLNs. The discrepancies between frozen section and permanent $\mathrm{H} \& \mathrm{E}$ evaluation are not unknown in the literature [30], and can reach as high as 27\% [27]. In the most recent study, Ballester et al. reported $56.3 \%$ sensitivity for intra-operative diagnostic methods [31]. For introduction of SLN mapping to any gynecological oncology center, this finding should be taken into account [32, 33].

Another important issue in the sentinel node mapping of midline tumors is the side of sentinel node identification [34, 35]. As midline tumors, lymphatic flow of the endometrial cancer should be bilateral in the pelvic area, however not all patients have bilateral lymphatic drainage during surgery. For example in our study, bilateral drainage could be detected in 16 out of 24 patients.

Bilateral drainage in midline tumors (including endometrial cancer) is very important. In case of sentinel node detection failure on one side, possibility of pathological lymph node involvement on the detection failure side should be considered. In other words, 
each patient is considered as two separate units and decision to continue to regional lymphadenectomy should be based on the pathological condition of sentinel nodes of each side separately. This strategy has proven to decrease the false negative rate of sentinel node mapping in midline tumors [36]. Our study had relatively low sample size and the effect of unilateral drainage on the accuracy of sentinel node mapping was not apparent.

\section{Conclusion}

Lymphatic mapping and sentinel node biopsy is feasible and accurate in endometrial cancer patients using combined radiotracer and blue dye methods. Frozen section accuracy was lower and underscores the importance of expert pathologists for SLN mapping technique.

\section{References}

1. Nayereh KG, Khadem G. Preventive and therapeutic vaccines against human papillomaviruses associated cervical cancers. Iran J Basic Med Sci 2012; 15: 585-601

2. Amant F, Moerman P, Neven P, Timmerman D, Van Limbergen E, Vergote I. Endometrial cancer. Lancet 2005; 366: 491-505.

3. Bouquier J, Bricou A, Delpech Y, Tulpin L, Akerman G, Barranger E. Is there an interest in para-aortic lymphadenectomy in operable endometrial cancer?. Bull Cancer 2010; 97: 199-209.

4. Bezu C, Coutant C, Ballester M et al. Ultrastaging of lymph node in uterine cancers. J Exp Clin Cancer Res 2010; 29: 5

5. Kang S, Lee JM, Lee JK et al. How low is low enough? Evaluation of various risk-assessment models for lymph node metastasis in endometrial cancer: a Korean multicenter study. J Gynecol Oncol 2012; 23: 251-256.

6. Abu-Rustum NR, Alektiar K, lasonos A et al. The incidence of symptomatic lower-extremity lymphedema following treatment of uterine corpus malignancies: a 12-year experience at Memorial Sloan-Kettering Cancer Center. Gynecol Oncol 2006; 103: 714-718.

7. Sadeghi R, Gholami H, Zakavi SR, Kakhki VR, Tabasi KT, Horenblas S. Accuracy of sentinel lymph node biopsy for inguinal lymph node staging of penile squamous cell carcinoma: systematic review and meta-analysis of the literature. J Urol 2012; 187: 25-31.

8. Sadeghi R, Tabasi KT, Bazaz SM et al. Sentinel node mapping in the prostate cancer. Meta-analysis. Nuklearmedizin 2011; 50: 107-115.

9. Glumac N, Hocevar M, Zadnik V, Snoj M. Inguinal or inguino-iliac/obturator lymph node dissection after positive inguinal sentinel lymph node in patients with cutaneous melanoma. Radiol Oncol 2012; 46: 258-264.

10. Suh DH, Kim K, Kim JW. Major clinical research advances in gynecologic cancer in 2011. J Gynecol Oncol 2012; 23: 53-64.

11. Sawicki S, Kobierski J, Lapinska-Szumczyk S et al. Comparison of SPECT-CT results and intraoperative detection of sentinel lymph nodes in endometrial cancer. Nucl Med Commun 2013.

12. Momennezhad M, Zakavi SR, Dabbagh Kakhki VR, Jangjoo A, Ghavamnasiri MR, Sadeghi R. Scatterogram: a method for outlining the body during lymphoscintigraphy without using external flood source. Radiol Oncol 2011; 45: 184-188

13. Aryana K, Gholizadeh M, Momennezhad M et al. Efficacy of high-energy collimator for sentinel node lymphoscintigraphy of early breast cancer patients. Radiol Oncol 2012; 46: 75-80.

14. Abu-Rustum NR, Khoury-Collado F, Pandit-Taskar N et al. Sentinel lymph node mapping for grade 1 endometrial cancer: is it the answer to the surgical staging dilemma? Gynecol Oncol 2009; 113: 163-169.

15. Niikura H, Okamura C, Utsunomiya $\mathrm{H}$ et al. Sentinel lymph node detection in patients with endometrial cancer. Gynecol Oncol 2004; 92: 669-674.
16. Sadeghi R, Forghani MN, Memar B et al. Comparison of pre-operative lymphoscintigraphy with inter-operative gamma probe and dye technique regarding the number of detected sentinel lymph nodes. Hell J Nucl Med 2009; 12: 30-32.

17. Jangjoo A, Forghani MN, Mehrabibahar $\mathrm{M}$ et al. Comparison of early and delayed lymphoscintigraphy images of early breast cancer patients undergoing sentinel node mapping. Nucl Med Commun 2010; 31: 521-525.

18. Buda A, Elisei F, Arosio M et al. Integration of hybrid single-photon emission computed tomography/computed tomography in the preoperative assessment of sentinel node in patients with cervical and endometrial cancer: our experience and literature review. Int J Gynecol Cancer 2012; 22: 830-835.

19. Cordero Garcia JM, Lopez de la Manzanara Cano CA, Garcia Vicente AM et al. Study of the sentinel node in endometrial cancer at early stages: preliminary results. Rev Esp Med Nucl 2012; 31: 243-248.

20. Kraft O, Havel M. Detection of Sentinel Lymph Nodes in Gynecologic Tumours by Planar Scintigraphy and SPECT/CT. Mol Imaging Radionucl Ther 2012; 21: 47-55

21. Echt ML, Finan MA, Hoffman MS, Kline RC, Roberts WS, Fiorica JV. Detection of sentinel lymph nodes with lymphazurin in cervical, uterine, and vulvar malignancies. South Med J 1999; 92: 204-208.

22. Holub Z, Kliment L, Lukac J, Voracek J. Laparoscopically-assisted intraoperative lymphatic mapping in endometrial cancer: preliminary results. Eur J Gynaecol Oncol 2001; 22: 118-121.

23. Ansari M, Rad MA, Hasanzadeh M et al. Sentinel node biopsy in endometrial cancer: systematic review and meta-analysis of the literature. Eur J Gynaecol Oncol 2013; 34: 387-401.

24. Barlin JN, Khoury-Collado F, Kim CH et al. The importance of applying a sentinel lymph node mapping algorithm in endometrial cancer staging: Beyond removal of blue nodes. Gynecol Oncol 2012; 125: 531-535.

25. Jangjoo A, Forghani MN, Mehrabibahar M, Sadeghi R. Anaphylaxis reaction of a breast cancer patient to methylene blue during breast surgery with sentinel node mapping. Acta Oncol 2010; 49: 877-878.

26. Ballester $M$, Dubernard $G$, Lecuru $F$ et al. Detection rate and diagnostic accuracy of sentinel-node biopsy in early stage endometrial cancer: a prospective multicentre study (SENTI-ENDO). Lancet Oncol 2011; 12: 469-476.

27. Solima E, Martinelli F, Ditto A et al. Diagnostic accuracy of sentinel node in endometrial cancer by using hysteroscopic injection of radiolabeled tracer. Gynecol Oncol 2012; 126: 419-423

28. Abu-Rustum NR, Gomez JD, Alektiar KM et al. The incidence of isolated paraaortic nodal metastasis in surgically staged endometrial cancer patients with negative pelvic lymph nodes. Gynecol Oncol 2009; 115: 236-238.

29. Kang S, Yoo HJ, Hwang JH, Lim MC, Seo SS, Park SY. Sentinel lymph node biopsy in endometrial cancer: meta-analysis of 26 studies. Gynecol Oncol 2011; 123: 522-527.

30. Memar B, Sadeghi R, Ayati NK et al. The value of touch imprint cytology and frozen section for intra-operative evaluation of axillary sentinel lymph nodes. Pol J Pathol 2010; 61: 161-165.

31. Ballester M, Dubernard G, Bats AS et al. Comparison of diagnostic accuracy of frozen section with imprint cytology for intraoperative examination of sentinel lymph node in early-stage endometrial cancer: results of Senti-Endo study. Ann Surg Oncol 2012; 19: 3515-3521.

32. Lax S, Tamussino K, Prein K, Lang P. Intraoperative frozen sections in diseases of the female genital tract. Pathologe 2012; 33: 430-440.

33. Baker P, Oliva E. A practical approach to intraoperative consultation in gynecological pathology. Int J Gynecol Pathol 2008; 27: 353-365.

34. Hassanzade M, Attaran M, Treglia G, Yousefi Z, Sadeghi R. Lymphatic mapping and sentinel node biopsy in squamous cell carcinoma of the vulva: systematic review and meta-analysis of the literature. Gynecol Oncol 2013; 130: 237-245.

35. Tehranian S, Treglia G, Krag DN et al. Sentinel node mapping in anal canal cancer: systematic review and meta-analysis. J Gastrointestin Liver Dis 2013; 22: 321-328.

36. Sadeghi R, Hasanzadeh M. Sentinel lymph node biopsy algorithm: can it be a universal method for midline tumors? Gynecol Oncol 2014; 132: 273-274. 